\title{
Archéobotanique des semences et des fruits de Lattara : bilan
} des recherches

Natàlia Alonso, Ramon Buxó, Núria Rovira, Isabelle Fauduet, Mme Isa OdenhardtDonvez

\section{Citer ce document / Cite this document :}

Alonso Natàlia, Buxó Ramon, Rovira Núria, Fauduet Isabelle, Odenhardt-Donvez Isa. Archéobotanique des semences et des fruits de Lattara : bilan des recherches. In: Gallia, tome 65, 2008. pp. 193-200;

doi : https://doi.org/10.3406/galia.2008.3342

https://www.persee.fr/doc/galia_0016-4119_2008_num_65_1_3342

Fichier pdf généré le 21/02/2020 


\title{
Résumé
}

Résumé. À travers plusieurs études systématiques concernant la fouille du site de Lattes, les analyses de semences et de fruits archéologiques ont permis de retracer l'évolution de la production, du traitement et de la consommation des végétaux entre la fin du premier âge du Fer et l'époque romaine (fin du Vle s. av. J.-C.-lle s. apr. J.-C.). Faisant valoir la richesse et les potentialités exceptionnelles du gisement, ce bilan de la recherche carpologique résume l'ensemble des données disponibles pour aborder les traits fondamentaux de l'alimentation végétale: acquisition ou cueillette, production ou agriculture. Les informations issues des espèces sauvages permettent de restituer les milieux végétaux fréquentés par les Lattarenses durant l'occupation de la ville antique.

\section{Zusammenfassung}

Zusammenfassung. Die Ausgrabungen in Lattes boten Gelegenheit Samen und Früchte aus den archäologischen Schichten zu analysieren und so die Entwicklung der Produktion, der Behandlung und des Verzehrs der Pflanzen vom Ende der älteren Eisenzeit bis zur römischen Periode (Ende 6. Jh. v. u. Z.-2. Jh. u. Z.) zurückzuverfolgen. Indem sie den Reichtum und die außergewöhnlichen Möglichkeiten des Fundplatzes geltend macht, fasst diese Bilanz der karpologischen Studien die Gesamtheit der zur Verfügung stehenden Daten zusammen, um die wesentlichen Züge der pflanzlichen Ernährung zur Sprache zu bringen: Erwerb oder Sammeln, Produktion oder Landwirtschaft? Darüber hinaus ermöglichen die Informationen, die sich aus der Untersuchung der Wildpflanzen ergeben, die von den Bewohnern des antiken Lattes frequentierten pflanzlichen Milieus zu rekonstruieren.

\begin{abstract}
Abstract. Through several systematic studies during the excavation of the site of Lattes, seed and fruit analyses made possible the determination of the evolution of plant production, process and consumption between the end of the early Iron Age and the Roman period (late 6th c. B. C.-2nd c. A. D.). This report on carpological research shows the resources and exceptional potentialities of the site. It summarizes the available informations to start on the dominant features of vegetable food, purchase or gathering activities, production or farming, and data taken from wild species allowing to reconstruct the botanical environment in which Lattarenses lived during the occupation of the antique town.
\end{abstract}




\title{
ARCHÉOBOTANIQUE DES SEMENCES ET DES FRUITS DE LATTARA
}

\author{
Bilan des recherches
}

\author{
Natàlia ALONSO, Ramon BUXó et Núria ROVIRA
}

Mots-clés. Âge du Fer, époque romaine, archéobotanique, carpologie, semences et fruits, France méridionale.

Résumé. À travers plusieurs études systématiques concernant la fouille du site de Lattes, les analyses de semences et de fruits archéologiques ont permis de retracer l'évolution de la production, du traitement et de la consommation des végétaux entre la fin du premier âge du Fer et l'époque romaine (fin du VIe s. av. J.-C.-II s. apr. J.-C.). Faisant valoir la richesse et les potentialités exceptionnelles du gisement, ce bilan de la recherche carpologique résume l'ensemble des données disponibles pour aborder les traits fondamentaux de l'alimentation végétale : acquisition ou cueillette, production ou agriculture. Les informations issues des espèces sauvages permettent de restituer les milieux végétaux fréquentés par les Lattarenses durant l'occupation de la ville antique.

Key-words. Iron Age, Roman period, archaeobotany, carpology, seeds and fruits.

Abstract. Through several systematic studies during the excavation of the site of Lattes, seed and fruit analyses made possible the determination of the evolution of plant production, process and consumption between the end of the early Iron Age and the Roman period (late $6^{\text {th }}$ c. B.C. $-^{\text {nd }}$ c. A.D.). This report on carpological research shows the resources and exceptional potentialities of the site. It summarizes the available informations to start on the dominant features of vegetable food, purchase or gathering activities, production or farming, and data taken from wild species allowing to reconstruct the botanical environment in which Lattarenses lived during the occupation of the antique town.

Translation: Isabelle FAUDUET

Schlüsselwörter. Eisenzeit, römische Zeit, Archäobotanik, Karpologie, Samen und Früchte.

Zusammenfassung. Die Ausgrabungen in Lattes boten Gelegenheit Samen und Früchte aus den archäologischen Schichten zu analysieren und so die Entwicklung der Produktion, der Behandlung und des Verzehrs der Pflanzen vom Ende der älteren Eisenzeit bis zur römischen Periode (Ende 6. Jh. v.u.Z.-2. Jh. u.Z.) zurückzuverfolgen. Indem sie den Reichtum und die außergewöhnlichen Möglichkeiten des Fundplatzes geltend macht, fasst diese Bilanz der karpologischen Studien die Gesamtheit der zur Verfügung stehenden Daten zusammen, um die wesentlichen Züge der pflanzlichen Ernährung zur Sprache zu bringen: Erwerb oder Sammeln, Produktion oder Landwirtschaft? Darüber hinaus ermöglichen die Informationen, die sich aus der Untersuchung der Wildpflanzen ergeben, die von den Bewohnern des antiken Lattes frequentierten pflanzlichen Milieus zu rekonstruieren.

Übersetzung: Isa ODENHARDT-DONVEZ

Des prélèvements et des analyses archéobotaniques de semences et de fruits archéologiques sont réalisés de façon systématique sur le site de Lattara depuis plus de vingt ans. Plusieurs études ont déjà été publiées, principalement dans la série Lattara (Buxó, 1989, 1991, 1992, 1996a, 1999, 2003 et 2005 ; Buxó et al., 1996), mais aussi dans d'autres revues scientifiques (Py, Garcia, 1993 ; Buxó, 1996b et 1997 ; Py,
Buxó, 2001). Les données issues des niveaux du V $\mathrm{V}^{\mathrm{e}} \mathrm{s}$. av. J.-C. seront prochainement publiées, quelques-unes ayant déjà été exposées dans les rapports de fouille (Alonso, 2003). Des informations provenant des alentours du site, correspondant à des chronologies antérieures mais aussi synchrones de la séquence de la ville, sont également disponibles grâce à l'étude du site de Port Ariane (Alonso et al., 2007). 


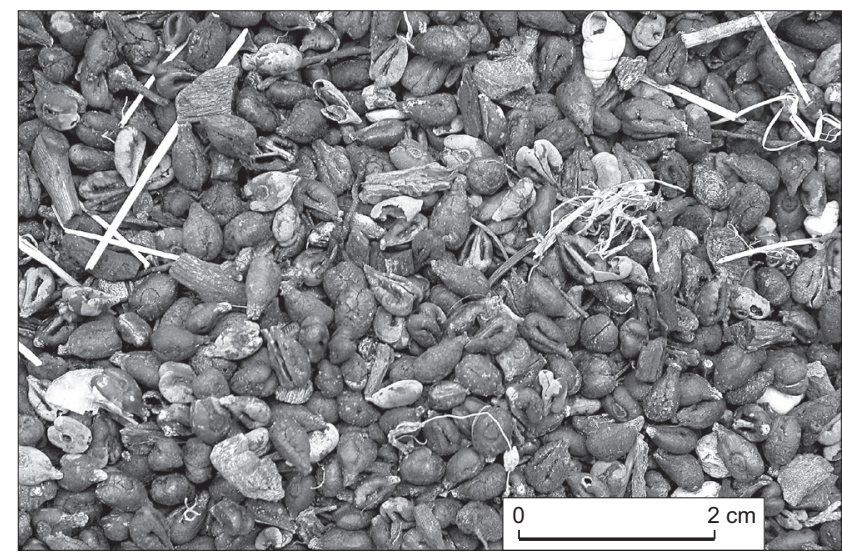

Fig. 127 - Grands amas de pépins de raisin (Vitis vinifera) provenant du puits PT615 de l'îlot 15 de Lattara (Us 15013) (cliché: R. Buxó, Musée archéologique de Catalogne).

La réalisation de nombreux tests expérimentaux, afin d'évaluer la présence et la fréquence des restes organiques dans les couches d'habitat, a permis de mettre au point une stratégie d'échantillonnage adaptée aux conditions de conservation du gisement. Les bases de cette méthodologie ont été largement diffusées, essentiellement dans les $n^{\text {os }} 2$ (Buxó, 1989), 4 (Buxó, 1991) et 5 (Buxó, 1992) de la série Lattara.

Les matériaux archéobotaniques analysés correspondent à des restes de semences et de fruits conservés dans les sédiments, principalement par carbonisation, mais aussi par minéralisation et, dans certains contextes, par imbibition. La plupart des carpo-restes ont été collectés de façon aléatoire dans des couches urbaines de remblai, de circulation ou de sédimentation de sol, surtout dans des niveaux à forte teneur en matière organique. Néanmoins, quelques concentrations ont également été décelées dans des ensembles particuliers correspondant à des structures isolées, comme les puits et les fosses, ainsi que des structures de combustion (foyers, fours) ou leurs vidanges (fig. 127).

\section{ASPEGTS FONDAMENTAUX SUR L'AGRICULTURE ET LA CONSOMMATION DE PRODUITS VÉGÉTAUX}

Les données archéobotaniques concernant le site de Lattara sont désormais très abondantes. La longue occupation de cette ville portuaire permet de connaître l'évolution de la présence et de l'usage des végétaux depuis la fin du premier âge du Fer jusqu'à l'époque romaine (fin du $\mathrm{VI}^{\mathrm{e}} \mathrm{s}$. av. J.-C.-II ${ }^{\mathrm{e}}$ s. apr. J.-C.).

\section{LES GULTURES CÉRÉALIÈRES}

Pendant toute la période d'occupation de la ville, on note la présence de nombreuses plantes cultivées, dont 7 céréales. La répartition par siècle des taxons de ce groupe montre que les cultures céréalières sont toujours présentes dans l'ensemble des plantes exploitées (tabl. IV). À toute époque, deux espèces sont les plus fréquentes et les plus abondantes, constituant certainement l'une des bases alimentaires majeures de la population : l'Orge vêtue et le Blé tendre/dur.

Des différences dans les proportions entre ces deux espèces ont été observées au cours des siècles. Cependant, elles ne peuvent pas être considérées comme un témoin direct des pratiques agricoles ou des modes de consommation des habitants, parce que, comme on l'a signalé ci-dessus, les prélèvements sont issus généralement de couches dispersées et non d'ensembles clos.

L'espèce la plus fréquente à toute époque est l'Orge vêtue (Hordeum vulgare), spécialement dans les couches les plus anciennes ( $\mathrm{V}^{\mathrm{e}}-\mathrm{III}{ }^{\mathrm{e}}$ s. av. J.-C.) et dans les concentrations. Dans la plupart des cas, ces restes n'ont pas conservé les glumes adhérées aux grains, bien que parfois quelques restes de glumelles et des glumes fragmentées, ainsi que des segments de rachis, aient été collectés.

Avec l'Orge vêtue, le Blé tendre/dur (Triticum aestivum/ durum) est la deuxième céréale en ordre de fréquence. Très peu de bases d'épillet ou de segments de rachis ont été collectés, ce qui laisse penser que les grains sont arrivés nettoyés en ville, prêts pour la consommation. Les caractéristiques morphométriques de certains caryopses de blé nu attestent un type d'épi compact (Triticum aestivum/durum type compactum), présent dans presque toute la séquence.

La fréquence d'apparition de l'Amidonnier (Triticum dicoccum) est élevée, mais loin derrière le Blé tendre/dur, le nombre de spécimens attestés étant très inférieur. En plus des grains, la présence de restes de battage (notamment des bases d'épillets) atteste sans doute des nettoyages dans le cadre domestique. L'Engrain (Triticum monococcum), en revanche, apparaît comme une espèce mineure, attestée uniquement de façon ponctuelle.

Le Millet commun (Panicum miliaceum) et le Millet italien (Setaria italica) n'ont été relevés que durant le second âge du Fer $\left(\mathrm{V}^{\mathrm{e}}-\mathrm{III}^{\mathrm{e}} \mathrm{s}\right.$. av. J.-C. $)$. Le nombre de spécimens collectés est réduit, mais la fréquence d'apparition est constante et croissante. L'absence de restes durant la période romaine pourrait être la conséquence d'une conservation 
Tabl. IV - Présence des taxons de plantes cultivées ou cueillies pendant chaque siècle de l'occupation de la ville de Lattara (cliché : R. Buxó, Musée archéologique de Catalogne).

\begin{tabular}{|c|c|c|c|c|c|c|c|}
\hline \multirow{2}{*}{\begin{tabular}{|ll} 
& TAXON ATtESTÉ \\
Céréales &
\end{tabular}} & \multicolumn{6}{|c|}{ Datation } & \multirow[b]{2}{*}{ Nom соммuN } \\
\hline & $\begin{array}{c}\text { V's. } \\
\text { av. J.-C. }\end{array}$ & $\begin{array}{c}\text { IV }{ }^{\mathrm{e}} \text { s. } \\
\text { av. J.-C. }\end{array}$ & $\begin{array}{c}\text { III' s. } \\
\text { av. J.-C. }\end{array}$ & $\begin{array}{c}\|^{\mathrm{e}} \mathrm{s} . \\
\text { av. J.-C. }\end{array}$ & $\begin{array}{c}\text { Ier }^{\text {s. }} \\
\text { av. J.-C. }\end{array}$ & $\begin{array}{c}\text { Ier s. } \\
\text { apr. J.-C. }\end{array}$ & \\
\hline Hordeum vulgare & $\bullet$ & $\bullet$ & $\bullet$ & $\bullet$ & $\bullet$ & $\bullet$ & Orge vêtue \\
\hline Triticum aestivum/durum & $\bullet$ & $\bullet$ & $\bullet$ & $\bullet$ & $\bullet$ & $\bullet$ & Blé tendre/dur \\
\hline Triticum aestivum/durum type compactum & $\bullet$ & $\bullet$ & $\bullet$ & $\bullet$ & $\bullet$ & & Blé tendre/dur de type compacte \\
\hline Triticum dicoccum & $\bullet$ & $\bullet$ & $\bullet$ & & $\bullet$ & • & Amidonnier \\
\hline Triticum monococcum & $\bullet$ & $\bullet$ & $\bullet$ & $\bullet$ & & & Engrain \\
\hline Panicum miliaceum & $\bullet$ & $\bullet$ & $\bullet$ & & & & Millet commun \\
\hline Setaria italica & $\bullet$ & $\bullet$ & & & & & Millet italien \\
\hline Avena sp. & $\bullet$ & $\bullet$ & $\bullet$ & $\bullet$ & & & Avoine \\
\hline \multicolumn{8}{|l|}{ Légumineuses } \\
\hline Cicer arietinum & & $\bullet$ & & & & & Pois chiche \\
\hline Lathyrus cicera & & $\bullet$ & $\bullet$ & & & & Gesse cultivée \\
\hline Lathyrus sativus & $\bullet$ & $\bullet$ & & & & $\bullet$ & Gesse \\
\hline Lens culinaris & $\bullet$ & $\bullet$ & $\bullet$ & $\bullet$ & & $\bullet$ & Lentille \\
\hline Medicago cf. sativa & & $\bullet$ & & & & & Luzerne \\
\hline Pisum sativum & $\bullet$ & $\bullet$ & $\bullet$ & & & $\bullet$ & Pois \\
\hline Vicia faba var. minor & $\bullet$ & $\bullet$ & $\bullet$ & & & $\bullet$ & Fève \\
\hline Vicia ervilia & $\bullet$ & $\bullet$ & $\bullet$ & & & & Ers \\
\hline Vicia sativa & & $\bullet$ & & & & & Vesce \\
\hline \multicolumn{8}{|l|}{ Oléagineuses } \\
\hline Linum usitatissimum & & $\bullet$ & & & & & Lin cultivé \\
\hline Linum sp. & $\bullet$ & & & & & & Lin \\
\hline \multicolumn{8}{|l|}{ Fruits cultivés } \\
\hline Celtis sp. & & & & & & $\bullet$ & Micocoulier \\
\hline Coriandrum sativum & & $\bullet$ & & & & & Coriandre \\
\hline Ficus carica & $\bullet$ & $\bullet$ & & & & & Figuier \\
\hline Juglans regia & & & & & & $\bullet$ & Noyer \\
\hline Olea europaea var. europaea & $\bullet$ & $\bullet$ & $\bullet$ & & & $\bullet$ & Olivier \\
\hline Prunus domestica & & & & & & $\bullet$ & Prunier \\
\hline Prunus persica & & & & & & $\bullet$ & Pêcher \\
\hline Vitis vinifera & $\bullet$ & $\bullet$ & $\bullet$ & $\bullet$ & $\bullet$ & $\bullet$ & Vigne \\
\hline \multicolumn{8}{|l|}{ Fruits cueillis } \\
\hline Arbutus unedo & $\bullet$ & & & & & & Arbousier \\
\hline Cornus mas & $\bullet$ & & & & & & Cornouiller mâle \\
\hline Corylus avellana & $\bullet$ & $\bullet$ & $\bullet$ & & & $\bullet$ & Noisetier \\
\hline Crataegus sp. & & $\bullet$ & & & & & Aubépine, Épine blanche \\
\hline Pinus pinea & & & & & & $\bullet$ & Pin pignon \\
\hline Prunus avium/cerasus & $\bullet$ & $\bullet$ & & & & & Cerise douce/aigre \\
\hline Prunus avium & & & & & & $\bullet$ & Cerise douce \\
\hline Prunus cerasus & & & & & & $\bullet$ & Cerise aigre \\
\hline Prunus spinosa & & & $\bullet$ & & & $\bullet$ & Prunellier \\
\hline Pyrus communis/pyraster & $\bullet$ & & & & & & Pomme/Poire \\
\hline Quercus ilex/coccifera & & & & & & $\bullet$ & Chêne vert /Kermès \\
\hline Quercus sp. & $\bullet$ & $\bullet$ & $\bullet$ & & & & Chêne \\
\hline Rubus fruticosus & $\bullet$ & & & & & & Mûre \\
\hline Rubus idaeus & & $\bullet$ & & & & & Framboise \\
\hline Rubus sp. & & & & & & $\bullet$ & Mûre/Framboise \\
\hline Sambucus nigra & $\bullet$ & & & & & & Sureau noir \\
\hline
\end{tabular}


différentielle des taxons, plutôt que d'un changement dans l'exploitation agronomique ou dans la consommation (Buxó, 1992, p. 75).

L'Avoine cultivée (Avena sativa) fait défaut à Lattara. Certains spécimens du genre Avena sp., collectés de façon constante ( $\mathrm{V}^{\mathrm{e}}-\mathrm{II}^{\mathrm{e}}$ s. av. J.-C.), mais en faible nombre, pourraient appartenir à l'espèce cultivée. Cependant, l'absence de bases de glumelles inférieures, caractéristiques de l'espèce cultivée, ne permet pas de les identifier formellement comme telles.

\section{LA VITICULTURE}

La mise au jour de nombreux pépins de raisin (Vitis vinifera) dans les fouilles de Lattara a fait de la problématique sur l'origine et le développement de cette culture, ainsi que de la vinification, l'un des sujets les plus notoires des études archéobotaniques menées sur le site. Cet aspect a été largement développé dans des publications spécifiques (Buxó, 1992 et 1996b ; Py, Buxó, 2001).

Certaines caractéristiques de la morphologie, l'évolution biométrique, ainsi que les études statistiques, témoignent en faveur d'une attribution des pépins de Lattara à des spécimens cultivés. Les courbes évolutives montrent un essor très significatif de la présence de pépins de raisin sur le site, notamment à partir du $\mathrm{III}^{\mathrm{e}}$ s. av. J.-C., que ce soit par rapport au total des taxons collectés ou au groupe des céréales (Buxó, 1992 et 1996b).

En fait, des restes de raisins sont déjà courants sur le site dans les couches des $\mathrm{V}^{\mathrm{e}}$ s. et $\mathrm{IV}^{\mathrm{e}}$ s. av. J.-C., et connus aussi pour le premier âge du Fer sur le site voisin de Port Ariane (Alonso et al., 2007). À cette époque, ils sont majoritairement associés à des résidus d'activités domestiques et leur fréquence est faible, ce qui indiquerait plutôt une consommation directe des raisins comme fruit (frais ou secs). En revanche, outre de grands amas de pépins, des restes de baies et des pédicelles ont été également collectés dans des niveaux plus tardifs de la ville ( ${ }^{\mathrm{er}} \mathrm{s}$. av. J.-C.), ce qui témoigne de la présence de résidus de pressurage (moût). Ces restes viennent appuyer l'hypothèse d'une production locale de vin.

L'évolution de la production viticole sur le site de Lattara peut être restituée en trois phases (Buxó, 1992, p. 79 ; 1999, p. 532-533 ; 2005, p. 217 ; Py, Garcia, 1993, p. 52-53 ; Py, Buxó, 2001, p. 41) :

a) existence d'une viticulture régulière dès le début du $\mathrm{V}^{\mathrm{e}}$ s. av. J.-C. ;

b) expansion à partir de la fin du $\mathrm{III}^{\mathrm{e}}$ s. av. J.-C. ; c) culmination à la fin du $\mathrm{II}^{\mathrm{e}}$ s. et au $\mathrm{I}^{\mathrm{er}}$ s. av. J.-C., au moment où de vastes plantations de vigne sont attestées dans les environs de la ville (Daveau dir., 2007).

Le développement de la culture de la vigne par rapport à la céréaliculture implique un changement fondamental dans les pratiques agricoles des Lattarenses, compte tenu des différences qui existent dans le rendement des cultures (annuelles versus pluriannuelles) et dans les techniques agricoles associées. Toutefois, il est peu probable que la culture des céréales ait véritablement subi une régression, car il s'agissait d'une culture essentielle à la survie alimentaire de la communauté.

\section{LÉGUMINEUSES, FRUITIERS ET PLANTES TEXTILES}

En sus des céréales et de la vigne, 9 espèces de légumineuses, 7 de fruits cultivés et 16 de fruits sauvages cueillis ont été consommées par les Lattarenses. S'y ajoute le Lin (Linum usitatissimum), plante oléagineuse aussi bien que textile, uniquement attestée durant les phases anciennes (tabl. IV).

De nombreuses espèces de légumineuses cultivées sont présentes depuis le $\mathrm{V}^{\mathrm{e}} \mathrm{s}$. av. J.-C. La Lentille (Lens culinaris) est l'une des plus fréquentes ; elle est représentée durant toute la séquence, à l'exception du I ${ }^{\mathrm{er}}$ s. av. J.-C. (tabl. IV). Le Pois (Pisum sativum), la Fève (Vicia faba var. minor), la Gesse cultivée (Lathyrus sativus) et l'Ers (Vicia ervilia) sont régulièrement présents durant la plupart des périodes. En revanche, le Pois chiche (Cicer arietinum), la Gesse chiche (Lathyrus cicera), la Vesce (Vicia sativa) et la Luzerne (Medicago sativa) sont beaucoup moins bien représentées.

Les légumineuses constituent une part importante de l'alimentation végétale des habitants de Lattara (comme en témoignent les ensembles illustrés au $\mathrm{IV}^{\mathrm{e}} \mathrm{s}$. par la maison incendiée 105), mais il n'existe pas à ce jour sur le site des traces d'un véritable stockage de ces produits végétaux (Buxó et al., 1996, p. 390 ; Buxó, 2003, p. 213).

Pour leur part, les fruits cultivés n'ont qu'une faible représentation dans les couches protohistoriques (tabl. IV), bien qu'y soient attestées certaines espèces au cours de l'âge du Fer, comme le Raisin (Vitis vinifera), la Figue (Ficus carica) et l'Olive (Olea europaea).

La Coriandre (Coriandrum sativum) apparaît de façon ponctuelle au $\mathrm{IV}^{\mathrm{e}}$ s. av. J.-C.

La grande quantité de noyaux et de coques conservés par imbibition dans deux des puits du $\mathrm{I}^{\mathrm{er}}$ s. apr. J.-C. (fig. 128) a permis d'augmenter le nombre d'attestations des fruits 


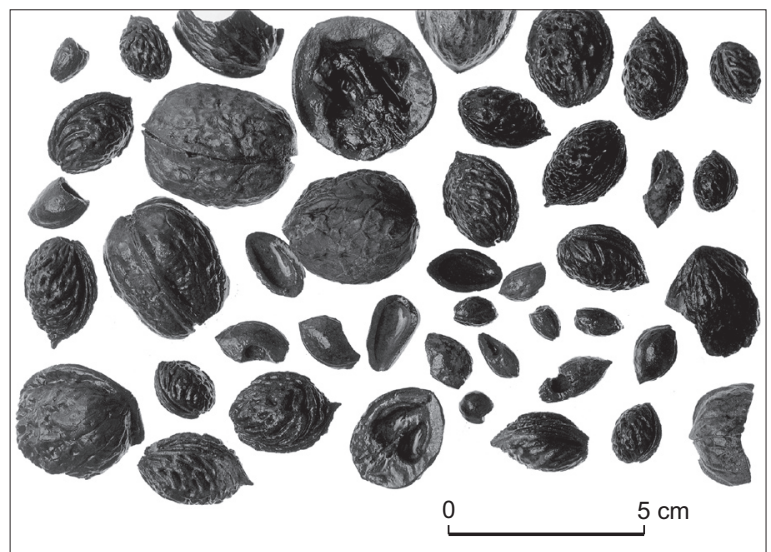

Fig. 128 - Échantillon de restes de fruitiers conservés par imbibition dans un puits de la zone 129 de Lattes (PT129011, Us 129024)

(cliché : R. Buxó, Musée archéologique de Catalogne).

cultivés et cueillis pour les périodes les plus récentes du site (Buxó, 2005). Des noix (Juglans regia), des noisettes (Corylus avellana), des pignons (Pinus pinea), des cerises douces (Prunus avium) et aigres (Prunus cerasus), deux variétés de prunes (Prunus domestica subsp. institia var. subrotunda, "prunes rondes ", et $P$. domestica subsp. institia var. juliana, prune crèque), des pêches (Prunus persica), des prunelles (Prunus spinosa), des raisins et des olives y ont été collectés.

Le nombre des restes d'olives n'est pas très élevé, mais elles sont présentes au moins depuis le début du $\mathrm{V}^{\mathrm{e}} \mathrm{s}$. av. J.-C. Divers facteurs peuvent être à l'origine de la sous-représentation de cette espèce : d'une part, le processus d'extraction de l'huile n'implique pas un contact direct avec le feu (ce qui diminue les chances de carbonisation) et, d'autre part, on doit envisager une possible réutilisation des résidus après le pressurage (Buxó, 2005, p. 217). La présence du bois d'olivier n'est pas identifiée sur le site avant l'époque romaine (voir Jorda, Chabal, Blanchemanche, supra, p. 11-21) ; il est possible que la culture de l'olivier n'ait pas eu une grande extension localement, et que l'on ait importé la plupart des produits sous une forme élaborée (huile et olives en saumure). Néanmoins, la découverte d'une maie de pressoir en position de fonctionnement dans une cour du $\mathrm{V}^{\mathrm{e}} \mathrm{s}$. (zone 27 : voir Belarte, supra, p. 105, fig. $76 \mathrm{n}^{\circ}$ 2) soulève la question des rapports existant entre les restes carpologiques ou anthracologiques de l'Olivier et sa culture/transformation.

Les fruits sauvages cueillis sont très variés durant toute la séquence d'occupation du site, jusqu'à un total de 16 taxons (tabl. IV). Ces espèces devaient faire partie de la végétation boisée, située éventuellement aux alentours $\mathrm{du}$ site, et témoignent d'une cueillette saisonnière de plusieurs ressources spontanées. Certaines de ces espèces (notamment le Prunellier ou les Cerisiers) ont pu bénéficier de soins particuliers à proximité des zones cultivées (Buxó, 1992, p. 71 et 2005, p. 216-217).

\section{CARACTÉRISTIQUES DES RESTES DE SEMENCES ET DE FRUITS EN CONTEXTES URBAINS SPÉCIFIQUES}

La plupart des restes de semences et de fruits ont été collectés, nous l'avons dit, de façon aléatoire dans divers types de couches. Cependant, dans quelques cas précis, des contextes spécifiques ont été échantillonnés, ce qui a permis une approche plus approfondie de la taphonomie ou de l'utilisation de certains végétaux.

\section{LES MAISONS INCENDIÉES}

L'échantillonnage effectué dans deux maisons détruites brutalement par un incendie au cours du IV s. av. J.-C. (la maison en torchis 104, mais surtout la maison 105 scellée par une toiture effondrée) a permis de connaître les produits végétaux stockés avant leur destruction, ainsi qu'un certain nombre de plantes sauvages. Ces dernières ont fourni des données importantes sur l'exploitation de plusieurs types de milieux végétaux (Buxó, 1996a ; Buxó et al., 1996 ; Roux, Chabal, 1996). Malheureusement, les espèces utilisées spécifiquement pour la construction de la toiture n'ont pas pu être isolées, car la variété taxonomique était très similaire à celle connue pour les contextes urbains généraux de la même époque (fig. 129).

\section{LES ESPACES OUVERTS : LA PLACE 123}

Cet espace, dont on a fouillé les niveaux des IV $^{\mathrm{e}}$-III ${ }^{\mathrm{e}}$ s. av. J.-C., a fait l'objet d'une monographie (Lattara, 16) dans laquelle les données carpologiques ont été étudiées en détail (Buxó, 2003). La densité moyenne de restes végétaux, ainsi que de taxons, attestés sur cette place, caractérise le cadre urbain de Lattara (fig. 129). Les deux types de couches identifiées (surfaces de circulation des hommes et du bétail et remblais constitués de déchets domestiques), ont fourni essentiellement des résidus provenant des habitations voisines. Toutefois, on peut envisager que certains de ces rejets soient liés à des activités réalisées sur place. La plupart des carpo-restes attestés sont des céréales à grain nettoyé (auxquelles se mêlent plusieurs adventices et rudérales), sans résidus de battage, correspondant à des rejets divers qui 


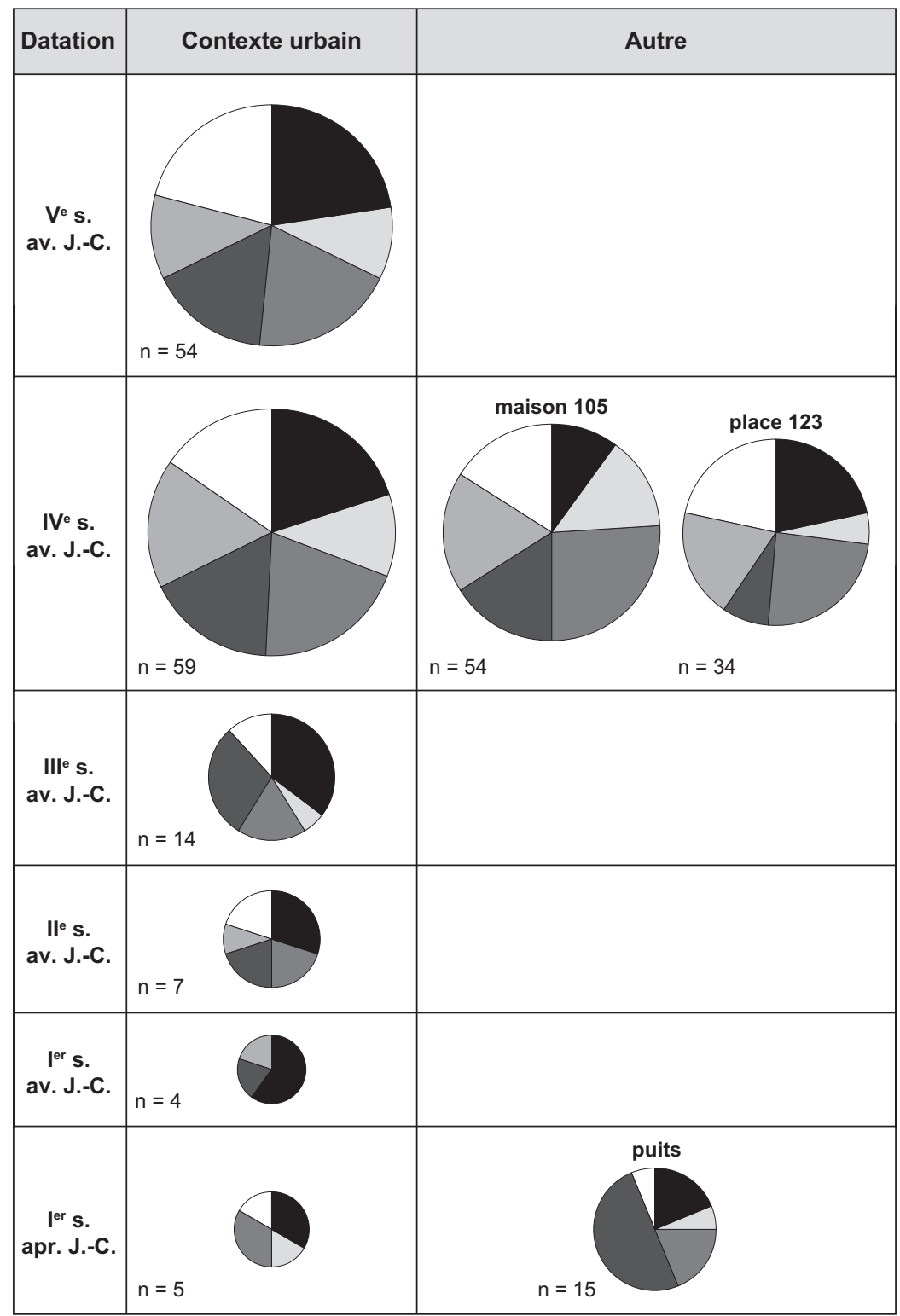

Plantes adventices: cultures céréalières

Adonis annua

Agrostemma githago

Asperula sp.

Avena sp.

Bromus sp.

Festuca sp.

Fumaria officinalis

Galium spurium

Heliotropium europaeum

Lolium temulentum

Phalaris paradoxa

Raphanus raphanistrum

Ridolfa segetum

Sherardia arvensis

Valerianella sp.

Veronica hederifolia

Plantes adventices: cultures sarclées

Amaranthus sp.

Atriplex hastata/patula

Carex divisa

Chenopodium album

Chenopodium polyspermum

Euphorbia helioscopia

Lathyrus sp.

Lithospermum arvense

Setaria sp.

Solanum nigrum

Plantes rudérales et des décombres

Amaranthus sp.

Bromus sp.

Carthamus sp.

Cerastium sp.

Chrysanthemum sp.

Galium aparine

Malva sylvestris

Poa annua

Polygonum aviculare

Polygonum convolvulus

Rumex crispus

Rumex pulcher

Sambucus ebulus

Silene alba
Haies, lisières,

ourlets forestiers, forêt

Arbutus unedo

Cornus mas

Corylus avellana

Crataegus sp.

Prunus spinosa

Quercus ilex/coccifera

Reseda lutea

Rosmarinus sp

Rubus sp.

Sambucus nigra

Silene dioica

Silene nutans

Solanum dulcamara

Trigonella sp.

Trinia glauca

Vicia sp.

Marais, roselières

Carex (espèces diverses)

Cladium mariscus

Cyperus sp.

Elatine hidropiper

Juncus sp.

Polygonum hydropiper Polygonum lapathifolium

Polygonum persicaria

Schoenus nigricans

Scirpus littoralis

Scirpus maritimus

Sparganium erectum

Prairies, pâturages

Asperula sp.

Centaurea sp.

Lolium perenne/rigidum

Luzula multiflora

Medicago lupulina

Medicago minima

Plantago lanceolata

Potentilla sp.

Rumex acetosella

Silene otites

Silene vulgaris

Thymelaea sp.

Trifolium $\mathrm{sp}$

Verbena officinalis

Fig. 129 - Comparaison de la fréquence relative des communautés végétales de plantes sauvages attestées à Lattara à partir du nombre de taxons par siècle $(n=$ nombre de taxons identifiés) et des taxons végétaux les plus représentatifs de chaque communauté (DAO : N. Alonso, Université de Lérida).

ont été éliminés dans les structures de combustion lors des préparations culinaires. Certains de ces rejets ont pu aussi constituer, de manière involontaire, un complément alimentaire pour les animaux présents dans l'espace.

\section{LES PUITS}

Cinq puits et leur comblement ont également fait l'objet d'une monographie récente (Lattara, 18) et d'une étude archéobotanique spécifique (Buxó, 2005). Ces données ont comblé de façon significative les lacunes existant dans la connaissance de la période romaine. Les comblements ont livré des restes carbonisés (céréales, légumineuses et quelques pépins de raisin), minéralisés (notamment de plantes sauvages) et surtout des restes imbibés d'eau (un éventail très large de fruits : voir supra, p. 196-197). Ces restes ont une origine alimentaire et correspondent probablement à des résidus domestiques. 


\section{LES OPÉRATIONS AGRICOLES ET L'EXPLOITATION DU MILIEU VÉGÉTAL}

Les données carpologiques de Lattara proviennent d'un milieu essentiellement urbain, ce qui impose une approche méthodologique et interprétative différente de celle qui pourrait être appliquée à un milieu rural. En raison du faible nombre de sous-produits agricoles collectés sur le site, et compte tenu des caractéristiques urbanistiques de la ville, on peut envisager que la plupart des opérations agricoles effectuées après la récolte aient été pratiquées à l'extérieur de la ville, dans des aires de battage situées probablement près des champs.

Dans le cadre de la ville, le grain a pu ne subir que des préparations directement liées à la consommation, comme le décorticage des grains vêtus (avec ou sans grillage), activité attestée par la présence de quelques glumes et glumelles dans plusieurs des échantillons prélevés. C'est le cas, par exemple, des ensembles de la maison en torchis 104 (IV s. av. J-C.), où des restes de battage de Blé amidonnier, Orge vêtue et Blé nu ont été collectés en association avec des grains stockés, de telle sorte que l'on peut envisager que ces céréales aient été apportées dans la maison avec leur épillet et transformées sur place juste avant la préparation de la nourriture (Buxó, 1996a).

Ces données sont compatibles avec la présence de plantes adventices, et même de rudérales, dans les bâtiments. En effet, les nettoyages quotidiens successifs ont dû créer des accumulations de restes, par exemple, dans le coin des murs ou aux alentours des foyers. En parallèle, la calcination extrême de nombreux microrestes témoigne probablement aussi de l'utilisation de déchets végétaux pour allumer les feux (Buxó, 1992, p. 77).

Les taxons appartenant aux plantes adventices (voir infra) montrent la présence majoritaire de semis de céréales d'automne (Orge et Blé tendre/dur), bien que des semis de printemps aient également été possibles (l'Avoine et aussi l'Orge). Les légumineuses auraient été pour la plupart semées au printemps, à l'exception de l'Ers ou de la Fève.

L'existence de mélanges intentionnels de différentes espèces dans une même parcelle est difficile à établir, compte tenu de l'absence d'ensembles clos et du fait que dans une récolte apparaissent des reliquats de plantes semées l'année (ou les années) précédente(s). Un mélange effectué après la récolte, lors d'un ensilage, ainsi que la possibilité de l'utilisation du méteil peuvent être cependant envisagés.
À la période antique, il est certain que la plaine s'étendant autour de Lattara était largement déboisée et utilisée pour les pratiques agricoles. Le développement de la culture de la vigne, qui vient s'ajouter vers le début du $\operatorname{III}^{\mathrm{e}} \mathrm{s}$. av. J.-C. aux cultures de céréales, implique une pression accrue sur le terroir (Chabal, 1991).

Ainsi, l'hypothèse d'une évolution progressive vers une vie plus urbaine et, par conséquent, plus éloignée des travaux agricoles, peut être posée, puisque la présence de restes de battage et de plantes adventices est plus fréquente et plus abondante dans les phases les plus anciennes. Cette hypothèse devra être réexaminée sur la base des données du $V^{\mathrm{e}}$ s. av. J.-C., actuellement à l'étude.

\section{LES PLANTES SAUVAGES ET L'ENVIRONNEMENT}

À l'heure actuelle, les analyses de semences et de fruits réalisées sur le site de Lattara ont permis d'identifier 114 taxons de plantes sauvages qui peuvent être regroupés par communautés végétales. Toutefois, il faut tenir compte du fait que certaines espèces peuvent faire partie de différents groupes, et de l'impossibilité occasionnelle d'identifier les taxons jusqu'à l'espèce, ce qui rend cette association peu précise.

D’une façon générale, les plantes sauvages attestées peuvent être classées dans cinq groupes principaux (fig. 129) :

Les plantes adventices : ce sont des semences arrivées sur le site mélangées à des produits végétaux divers, car les plantes poussent dans les mêmes champs. Deux groupes sont représentés : les plantes qui accompagnent les céréales d'hiver et celles, plus caractéristiques, des semis de printemps ou des terrains sarclés. Le premier groupe est composé de 26 taxons associés à des terrains calcicoles et à des endroits chauds et ensoleillés (Buxó et al., 1996, p. 392). En revanche, le deuxième groupe est plutôt lié à des sols riches en substances azotées et caractérisés par des sarclages répétés.

Les plantes rudérales et des décombres : ces plantes colonisent les milieux bouleversés, comme les déblais, les endroits piétinés, comme les bords des chemins et les voies, ainsi que des lieux riches en matières organiques, comme les dépotoirs et les décombres. Un total de 24 taxons de ce groupe a été collecté sur le site. Ces plantes ont pu pousser dans les aires de circulation ou les dépotoirs de la ville, mais aussi arriver des alentours par voie animale ou même humaine.

Les plantes des haies, lisières et ourlets forestiers : situé dans la forêt, en lisière de forêt ou le long des haies, ce groupe concerne un total de 16 taxons, dont certains correspondant à des arbustes ou des arbres à fruits qui ont pu être cueillis et consommés par les Lattarenses. 
Les plantes des marais et roselières : ce type de végétation colonise essentiellement les bords des cours d'eau et les zones humides, mais certaines espèces peuvent se développer aussi dans les champs. Ce groupe est attesté à Lattara par 23 taxons. On a pu mettre en évidence le transport vers la ville de plusieurs de ces espèces en même temps que les roseaux des marais utilisés pour la construction de la toiture de la maison 105 (Buxó et al., 1996).

Les prairies et les pâturages : ce sont des formations artificielles destinées à l'élevage du bétail, certaines succédant régulièrement aux cultures. En parallèle, d'autres pourraient se localiser sur des sols secs ou ensoleillés, colonisant dans la ville des espaces ouverts créés par la pâture des ovicapridés (Buxó et al., 1996, p. 394-395). Un total de 16 taxons pouvant appartenir à ce groupe a été identifié.

\section{LES CONNAISSANCES ACTUELLES}

Jusqu'à présent, les résultats des études archéobotaniques menées sur l'agglomération urbaine de Lattara montrent une diversification progressive de la consommation de produits végétaux, depuis les périodes les plus anciennes jusqu'à l'époque romaine.

Au cours de la séquence étudiée, une exploitation de plus en plus centrée sur les céréales les plus productives (blés nus, orges vêtues), complétée par d'autres produits (autres céréales, légumineuses, fruits cultivés ou sauvages et plantes textiles/oléagineuses), peut être soulignée. Ces produits végétaux divers proviendraient essentiellement, soit des alentours immédiats de la ville, soit, par échange, des terroirs gérés par d'autres agglomérations ou des fermes environnantes. En parallèle, un commerce à plus longue distance, concernant des produits élaborés divers (par exemple, l'huile ou les olives), ainsi que certains fruits ou épices (coriandre ?), peut également être envisagé.
À partir du III ${ }^{\mathrm{e}}$ s. av. J.-C., on doit ajouter à la production principale d'Orge et de Blé tendre/dur celle du Raisin. La culture de la Vigne, bien qu'attestée depuis le $\mathrm{V}^{\mathrm{e}} \mathrm{s}$. av. J.-C., prend alors un véritable essor témoignant du développement de la viniculture locale.

À partir du milieu du IV ${ }^{\mathrm{e}}$ s. av. J.-C., les restes carpologiques (à l'exception des grands amas de pépins de raisin) se présentent presque toujours dispersés et mélangés en proportions variables. Le nombre de restes de battage ou de plantes adventices n'est pas très élevé. Ces faits pourraient suggérer une distribution en ville de produits déjà préconditionnés pour la consommation, surtout dans le cas de céréales nues comme le Blé tendre/dur. Il conviendra de vérifier si cette hypothèse peut être appliquée aux périodes antérieures, ou si au contraire une gestion agricole directe était plus répandue dans les premières phases de l'habitat.

L'expansion des cultures à la période romaine, qui entraîne la mise en valeur de nouveaux produits agricoles, peut clairement être considérée comme une inflexion dans les traditions de consommation végétale lattoises. À partir de l'époque augustéenne en effet, les menus s'enrichissent d'une multitude de fruits cultivés, comme en témoigne la diversité des espèces collectées dans les puits.

Un prélèvement systématique de milliers d'échantillons durant une vingtaine d'années, le traitement de grands volumes de sédiments, ainsi que la présence constante d'archéobotanistes sur le chantier de fouille, ont permis que Lattara soit le site du sud de la France le plus intensivement étudié. La longue séquence d'occupation de cette ville portuaire, qui s'étend de la fin du premier âge du Fer jusqu'à l'époque romaine, fait et continuera de faire de ce gisement un lieu privilégié pour l'étude des questions majeures concernant l'agriculture, la consommation et le commerce des végétaux au cours de la Protohistoire et de l’Antiquité méditerranéennes. 